\title{
BreastCore
}

\section{Immunological Mechanisms in Breast Cancer - from Bench to Bedside}

\author{
Carsten Denkert ${ }^{\mathrm{a}} \quad$ Florian Schütz $^{\mathrm{b}}$ \\ a Institute of Pathology and German Cancer Consortium (DKTK), Charité, Berlin, Germany; \\ ${ }^{b}$ Breast Cancer Center, University of Heidelberg, Heidelberg, Germany
}

Immunological parameters have been described as prognostic factors in many different tumor types, including colon cancer, malignant melanoma, and lung cancer, with promising results of clinical trials of immunomodulatory agents [1]. The upcoming immune therapies have the potential to transform the way malignant tumors are treated in many fields of oncology [2].

Traditionally, breast cancer has not been viewed as a particularly immunogenic type of tumor. Therefore, the first clinical trials of immune checkpoint inhibitors were focused on other types of tumors, such as malignant melanoma and non-small cell lung cancer. Nevertheless, immune parameters have been studied to a great extent in breast cancer [3] and accumulating data shows that they are relevant for the development and progression of this tumor type [4]. Consequently, immunotherapies of breast cancer are now tested in different clinical trials.

In this issue of BREAST CARE the immunogenicity of breast cancer is described from various points of view. From the point of view of immunologists, the relevance of immune checkpoints is described in normal biological processes as well as malignant disease [5]. From the pathologist's viewpoint, the analysis of tumor-infiltrating lymphocytes (TILs) offers a first approach to the correlation of different immunological patterns in breast cancer with therapy response, which can be further investigated by specific immunological markers [6]. From the viewpoint of the clinical oncologist, there is a variety of options to build therapeutic strategies on the increasing knowledge of the immunological mechanisms [7].

These new therapeutic options will - however - generate new challenges, for example in the field of molecular diagnostics [8]. In parallel to the new therapeutic approaches of immunotherapy, new diagnostic tests are needed. Not all patients will benefit from immunotherapy, and it will be essential to identify those patients that have the highest benefit as well as those patients that do not benefit from immunotherapy at all. From a diagnostic point of view, the immune system is particularly difficult to monitor, due to its high complexity and its high degree of spatial and temporal heterogeneity. There are different types of biomarkers as well as different technologies that could be used. Interestingly, one of the simplest approaches so far, the evaluation of TILs, has generated highly reproducible results on the relevance of immune parameters for response to neoadjuvant chemotherapy and patient prognosis, in particular in triple-negative breast cancer [9]. The evaluation of TILs can be performed using already existing H\&E slides from histopathological diagnosis as well as slides collected in the course of clinical studies. The International Immunooncology Working Group ('TIL Working Group') has published a first guideline for the standardized evaluation of TILs [10], and the retrospective evaluation of a multitude of existing clinical trial cohorts for TIL levels is currently in progress. Furthermore, it is planned to use TILs as a stratification parameter in clinical trials on immunotherapeutic agents in breast cancer. In addition to the relatively simple evaluation of TILs, molecular signatures have been described that might offer additional predictive power [11], in particular in those tumors with intermediate and heterogeneous TIL levels. In addition to the primary diagnostic approaches, it will be important to monitor the development of resistance to immunotherapy.

Over the last 20 years we have learned much about the interaction of solid tumors and the immune system. With this understanding has come a renaissance in cancer therapy, as immunotherapeutic interventions, which augment tumor-specific responses and block suppressive pathways on which tumors depend to maintain their immune privilege, have shown increasing efficacy in the clinic. However, despite the progress we have made in understanding these mechanisms we have just started to transfer this knowledge into therapeutic implications.

Trastuzumab was the first antibody that could induce an antigen-specific antitumor immune reaction [12]. Until today it remains unclear whether the main effect of trastuzumab is related to immunological mechanisms or to synergistic activity with chemotherapy [13]. Meanwhile, many antibodies were approved for treating solid tumors including breast cancer. However, antibodies represent only a small component of the immunotherapeutic repertoire.

\section{KARGER}

Fax +497614520714
() 2016 S. Karger GmbH, Freiburg

1661-3791/16/0112-0093\$39.50/0
Prof. Dr. med. Florian Schütz

Universitätsfrauenklinik Heidelberg

Interdisziplinäres Brustzentrum

Im Neuenheimer Feld 440, 69120 Heidelberg, Germany

florian.schuetz@ med.uni-heidelberg.de 
There are 3 different ways of immunotherapy: A. active vaccination, B. passive vaccination, C. immunomodulation. Active vaccination or immunization is defined as stimulation with a specific antigen to promote an antigen-specific immunoreaction in the body. In this special issue of BREAST CARE, Clifton and colleagues [14] give insight into their interesting research work about a vaccine against HER2-associated breast cancer. An immunogenic peptide (E75) derived from the HER2 protein has been given in combination with an immunoadjuvans to high-risk early breast cancer patients in a phase II trial. The 5-year disease-free survival was significantly higher in the vaccinated patient cohort in comparison to patients who received standard treatment. Meanwhile, the research group is doing a phase III trial in early breast cancer patients.

Passive vaccination is the transfer of active humoral immunity in the form of ready-made antibodies or effector lymphocytes, such as activated $\mathrm{T}$ cells. While antibodies are already in broad clinical routine, adoptive cellular immunotherapies are not well established yet.

Immunomodulation is defined as a modification of the immune response or the functioning of the immune system. Monoclonal antibodies, cytokines, glucocorticoids, immunoglobulins, ultraviolet light, plasmapheresis, or related agents known to alter cellular or humoral immunity can be used to manipulate the immune response. Here, T-cell checkpoint blockade was named 'Breakthrough of the Year' by Science magazine in 2013 because of its success in treating melanoma as well as lung cancer. Bedognetti and colleagues describe this treatment in this issue as being an option in breast cancer as well [5]. However, only the minority of breast cancer patients with metastatic disease has responded to an anti-PD-1 therapy with the antibody pembrolizumab (18\%). Extensive future research is urgently needed to identify those responders before starting the treatment. Whether a specific immune gene signature can be used is still under investigation but first results in other solid tumors look quite promising [15].

Immunotherapies will soon be standard of care in breast cancer patients but 'one size doesn't fit all' is a dictum that must be postulated for the wide range of immune treatments. We have to better define subpopulations of breast cancer patients to optimize the immunological way to attack tumor cells.

\section{Disclosure Statement}

The authors declare no conflicts of interest.

\section{References}

1 Postow MA, Callahan MK, Wolchok JD: Immune checkpoint blockade in cancer therapy. J Clin Oncol $2015 ; 33: 1974-1982$.

2 Khalil DN, Smith EL, Brentjens RJ, Wolchok JD: The future of cancer treatment: immunomodulation, CARs and combination immunotherapy. Nat Rev Clin Oncol 2016; DOI: 10.1038/nrclinonc.2016.25..

3 Kroemer G, Senovilla L, Galluzzi L, André F, Zitvogel L: Natural and therapy-induced immunosurveillance in breast cancer. Nat Med 2015;21(10):1128-1138.

4 Galluzzi L, Buqué A, Kepp O, Zitvogel L, Kroemer G Immunological Effects of Conventional Chemotherapy and Targeted Anticancer Agents. Cancer Cell 2015;28: 690-714.

5 Bedognetti D, Maccalli C, Al Bader SBJ, Marincola FM, Seliger B: Checkpoint inhibitors and their application in breast cancer. Breast Care 2016; DOI: 10.1159/ 000445335.

6 Ingold Heppner B, Loibl S, Denkert C: Tumor-infiltrating lymphocytes: a promising biomarker in breast cancer. Breast Care 2016; DOI: 10.1159/000444357.

7 Domschke C, Schneeweiss A, Stefanovic S, Wallwiener M, Heil J, Rom J, Sohn C, Schuetz F: Cellular Immune Responses and Immune Escape Mechanisms in Breast Cancer: Determinants of Immunotherapy. Breast Care 2016; DOI: $10.1159 / 000445061$.
- 8 Topalian SL, Taube JM, Anders RA, Pardoll DM: Mechanism-driven biomarkers to guide immune checkpoint blockade in cancer therapy. Nat Rev Cancer 2016 DOI: $10.1038 / \mathrm{nrc} .2016 .36$.

9 Savas P, Salgado R, Denkert C, Sotiriou C, Darcy PK, Smyth MJ, Loi S: Clinical relevance of host immunity in breast cancer: from TILs to the clinic. Nat Rev Clin Oncol 2016;13:228-241.

10 Salgado R, Denkert C, Demaria S, Sirtaine N, Klauschen F, Pruneri G, Wienert S, Van den Eynden G, Baehner FL, Penault-Llorca F, Perez EA, Thompson EA, Symmans WF, Richardson AL, Brock J, Criscitiello C, Bailey H, Ignatiadis M, Floris G, Sparano J, Kos Z, Nielsen T, Rimm DL, Allison KH, Reis-Filho JS, Loibl S, Sotiriou C, Viale G, Badve S, Adams S, Willard-Gallo K, Loi S; International TILs Working Group 2014: The evaluation of tumor-infiltrating lymphocytes (TILs) in breast cancer: recommendations by an International TILs Working Group 2014. Ann Oncol 2015;26:259-271
11 Denkert C, von Minckwitz G, Brase JC, Sinn BV, Gade S, Kronenwett R, Pfitzner BM, Salat C, Loi S, Schmitt WD, Schem C, Fisch K, Darb-Esfahani S, Mehta K, Sotiriou C, Wienert S, Klare P, André F, Klauschen F, Blohmer JU, Krappmann K, Schmidt M, Tesch H, Kümmel S, Sinn P, Jackisch C, Dietel M, Reimer T, Untch M, Loibl S: Tumor-infiltrating lymphocytes and response to neoadjuvant chemotherapy with or without carboplatin in human epidermal growth factor receptor 2-positive and triple-negative primary breast cancers. J Clin Oncol 2015;33:983-991.

12 Slamon DJ1, Leyland-Jones B, Shak S, Fuchs H, Paton V, Bajamonde A, Fleming T, Eiermann W, Wolter J, Pegram M, Baselga J, Norton L: Use of chemotherapy plus a monoclonal antibody against HER2 for metastatic breast cancer that overexpresses HER2. N Engl J Med 2001;344:783-792

13 Moasser MM: Two dimensions in targeting HER2. J Clin Oncol 2014;32:2074-2077.

14 Clifton GT, Gall V, Peoples GE, Mittendorf EA: Clinical development of the E75 vaccine in breast cancer. Breast Care 2016; DOI: 10.1159/000446097.

15 Seiwert TY, et al.: Inflamed-phenotype gene expression signatures to predict benefit from the anti-PD-1 antibody pembrolizumab in PD-L1+ head and neck cancer patients. J Clin Oncol 2015;33suppl: abstr. 6017. 